\title{
Worrisome outbreak of river blindness in northern Uganda
}

A lthough the number of cases of onchocerciasis, commonly known as river blindness, is declining in most of Africa, a worrisome upsurge is being recorded in northern Uganda along the southern Sudan border, where conflict has reigned for most of the last 2 decades.

Richard Ndyomugyenyi, head of the Ugandan program that fights the disease, says that while overall infection rates in the country have declined in recent years, higher infection rates were recorded in parts of northern Uganda in the latter half of 2009.

The remote areas, such as Kitgum and Pader districts, are in a part of Uganda where the Lord's Resistance Army rebels have operated for 18 years, making them inaccessible to health interventions, Ndyomugyenyi says.

River blindness is a disease caused by Onchocerca volvulus, a parasitic worm transmitted to humans by the bite of infected black flies. Worm larvae that are transmitted develop into adult worms that settle in fibrous nodules on the human body, often causing such effects as intense itch, depigmentation of the skin and elephantiasis of the genitals. When the worms reach the cornea, they can cause visual impairment or blindness. The disease is treated with the drug ivermectin, which kills the microfilariae, although, in 2008, some researchers reported that adult worms were resistant to the drug.

The number of people infected with onchocerciasis stands at 1.5 million in Uganda, government statistics indicate. International programs have largely eliminated river blindness as a major public health problem in West African countries such as Benin, Burkina Faso, Ghana, Guinea Bissau, Guinea, Ivory Coast, Mali, Niger, Senegal, Sierra Leone and Togo.

Still, the World Health Organization says more than 17 million people remain infected with onchocerciasis, almost all (99\%) in Africa. The disease is most endemic in Nigeria and regions within

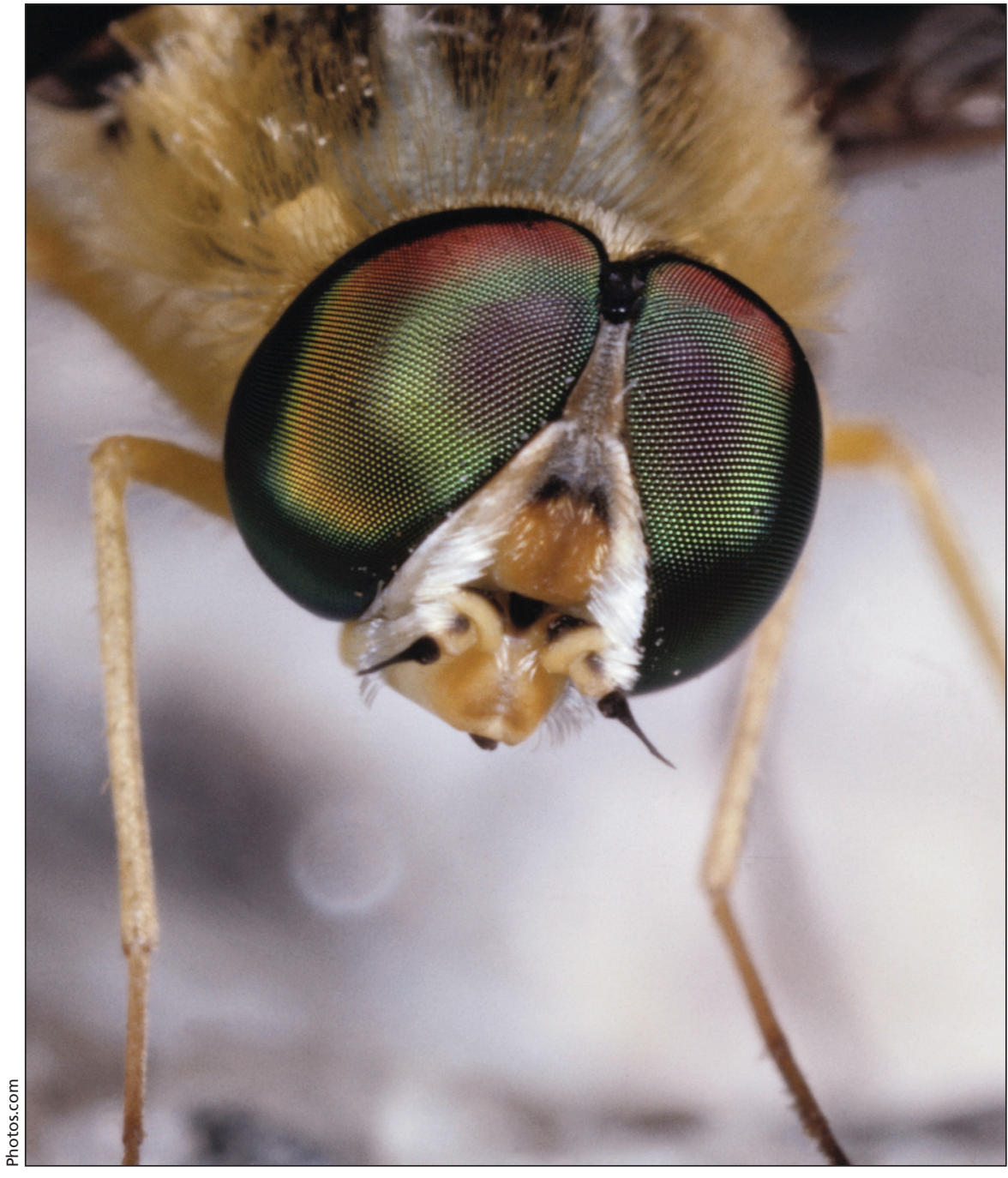

Onchocerciasis is caused by a parasitic worm that is transmitted to humans by infected black flies.

Cameroon, Ethiopia, Sudan, and Uganda.

Over the last 3 years, Sudan and Uganda have undertaken a twice-a-year elimination strategy modelled on an approach used to eliminate the disease in Central and South America. The Americas approach essentially involved administering ivermectin every 6 months to $85 \%$ of people infected with onchocerciasis in 6 endemic countries: Brazil, Colombia, Ecuador, Guatemala, Mexico and Venezuela.

But while the approach has helped reduce transmission rates in parts of
Uganda, the shortage of health workers and difficulties in accessing some remote areas remain challenges, says Uche Amazigo, who has for more than a decade led the African Programme for Onchocerciasis Control.

Over the course of the past 3 decades, the Canadian International Development Agency has contributed over $\$ 30$ million to international river blindness eradication initiatives. Wairagala Wakabi, Kampala, Uganda

DOI:10.1503/cmaj.090821 